\title{
Subacute dementia and imaging correlates in a case of Fahr's disease
}

\author{
T Benke, E Karner, K Seppi, M Delazer, J Marksteiner, E Donnemiller
}

J Neurol Neurosurg Psychiatry 2004;75:1 163-1165. doi: 10.1136/jnnp.2003.019547

\begin{abstract}
We report a case of idiopathic bilateral basal ganglia calcinosis, or Fahr's disease (FD) in a 50 year old patient who developed rapidly progressive behavioural abnormalities and severe neuropsychological impairments, but no movement disorder. Neuropsychological deficits included a severe dysexecutive syndrome, anterograde amnesia, and attentional impairment. Neuropsychiatric features comprised apathy with intermittent disinhibition, anxiety, irritability, frequent mood changes, ritualistic and antisocial behaviour, and psychosis. Fluorodeoxyglucose positron emission tomography showed a massive reduction of glucose metabolism in the basal ganglia and the frontal brain. The observed abnormalities possibly result from a disruption of frontostriatal circuits, presumably at the basal ganglia level. This case indicates that FD may cause exclusively behavioural alterations and that the associated hypometabolism in certain frontal areas is closely related to the clinical picture.
\end{abstract}

$\mathrm{F}$ ahr's disease (FD) refers to a sporadic or familial idiopathic calcification of the basal ganglia that may lead to neurological, psychiatric, and cognitive abnormalities. Patients with FD mostly present with movement disorders such as parkinsonism, chorea, tremor, dystonia, dysarthria, paresis, or speech impairment. Other common neurological features are seizures, syncope, or stroke-like events, often combined with a frontal subcortical pattern of behavioural dysfunction and psychiatric symptoms such as psychosis, mood disorders, and dementia. ${ }^{12}$ The origin and pathomechanism of this disorder are unknown, as is the reason why other cases with basal ganglia calicification remain asymptomatic. ${ }^{3}$ Fluoro-L-dopa uptake was found normal in a study of FD patients, suggesting an intact nigrostriatal dopaminergic pathway. ${ }^{4}$ However, two single case studies have found reduced focal cerebral blood flow and glucose metabolism ${ }^{5}{ }^{6}$, thus the observed symptoms may be manifestations of a cortico-subcortical disconnection. It is therefore of special interest to determine which parts of the brain show reduced metabolism in FD, and whether areas of hypometabolism correspond with the clinical picture. The present report documents neurobehavioural and imaging data in parallel in order to further analyse the relationship between behavioural, structural, and metabolic findings in FD.

\section{CASE REPORT}

A 50 year old, previously healthy man presented with a 6 month history of personality change and an impairment of planning and memory functions. He had led an active and successful life as a general practitioner until he rapidly became unable to continue his work because of cognitive deficits, attention problems, irritability, mood changes (dysphoria alternating with episodes of constricted affect and anxiety), and ritualised behaviours. The patient had no history of alcohol or drug abuse. The family history was unremarkable regarding dementia or FD. His neurological examination was normal, as was his orientation and language. A neuropsychological investigation showed a severe impairment of memory functions, verbal more than figural, and reduced psychomotor speed and verbal associative skills (table 1), but no ideomotor apraxia, dysarthria, or visuoconstructive impairment. On a computerised test battery measuring alertness, divided attention, and the suppression of irrelevant stimuli, ${ }^{7}$ the patient's reaction times were drastically slowed and he committed many errors. CT and MRI showed bilateral calcifications in the basal ganglia, mostly in the pallidum, but there was no frontal or temporal atrophy. Brain 18-fluorodeoxyglucose positron emission tomography (FDG-PET) was performed with an Advance PET scanner (General Electric Medical Systems, Milwaukee, USA), and showed a massive reduction of glucose metabolism in the basal ganglia and both frontal lobes (fig 1). Laboratory and endocrinological investigations were all normal. In particular, screening for hypoparathyroidism, iron or copper deficiencies, mitochondrial encephalopathies, encephalitis, and Creutzfeld-Jakob disease were all negative, and serum and urine calcium metabolism was normal. No thyroid disease or vitamin deficiency could be found. The EEG was normal.

Twelve months after disease onset, the patient's cognitive and behavioural functions had further deteriorated. Learning and spontaneous recall of a 16 item word list had further declined, and he made many errors of committment and perseverations during memory tests. The most striking deficits were found on tasks measuring executive functions. Owing to continuous perseverations and rule breaking behaviour, he was unable to perform the Action Program Test, a task requiring simple, practical, problem solving abilities. ${ }^{8}$ On the "odd man out" choice discrimination task, in which subjects have to alternate between two different rules, his set shifting abilities appeared completely eradicated. ${ }^{9}$ Similarly, suppression of irrelevant stimuli, as demanded in a simple interference naming task, was impossible. Verbal fluency was grossly impaired. Timed tests of attention including alertness, and divided and selective attention showed markedly slowed reaction times or could not be performed (go/no go task; table 1). His behaviour was now characterised by apathy, intermittent disinhibition, distractability, poor impulse control, increasing antisocial behaviour and physical aggression, compulsions and anxiety. $\mathrm{He}$ also lost all insight into his condition. External rating by his wife on the Neuropsychiatric Inventory ${ }^{10}$ showed particular elevation of the subscales agitation/aggression,

Abbreviations: FD, Fahr's disease; FDG-PET, fluorodeoxyglucose positron emission tomography 
Table 1 Neuropsychological results (percentage scores in brackets)

\begin{tabular}{lll}
\hline Months after disease onset & 7 & 12 \\
\hline Estimated premorbid IQ & & $107(75)$ \\
Digit Span & fw $6(13)$, & fw $5(2)$, \\
& bw $2(<2)$ & $4(2)$ \\
California Verbal Learning Test & & \\
$\quad$ Verbal learning, Trial 1-5 & $5-7-8-7-8$ & $4-5-6-7-7$ \\
& $(<2)$ & $(<2)$ \\
Short-delay free, cued recall & $4,6(<2)$ & $6,4(<2)$ \\
Long-delay free, cued recall & $7,7(<2)$ & $6,6(<2)$ \\
Recognition hits, false positives & $15,2(<16)$ & $16,12(<2)$ \\
Intrusions & 0 & 6 \\
$\quad$ Perseverations & 16 & 14 \\
Rey-Osterrieth Complex Figure Test & & \\
Copy & $32(10)$ & $25(<1)$ \\
Immediate recall & $17.5(27)$ & $20.5(62)$ \\
Delayed recall & $17(21)$ & $19.5(54)$ \\
Recognition & $21(54)$ & $16(<1)$ \\
Trail Making Test, Form A (s) & $38(25-50)$ & $38(25-50)$ \\
Trail Making Test, Form B (s) & $168(<10)$ & Failed \\
Word list generation & $7(<10)$ & $6(<10)$ \\
Animals/min & $7(<10)$ & $2(<10)$ \\
S-words & & \\
Test for Attentional Performance & $418(1)$ & $485(<1)$ \\
Alertness, no warning tone (ms) & $378(1)$ & $421(1)$ \\
Alertness, warning tone (ms) & $926(1)$ & Failed \\
Divided attention (ms) & $703(1)$ & Failed \\
Go/no go (ms) & & \\
\hline *Maximum score= 16. & & \\
\hline
\end{tabular}

irritability/lability, disinhibition, and dysphoria. The Frontal Systems Behavior Scale, ${ }^{11}$ a validated scale designed to measure behaviours associated with frontal lobe damage based on family ratings, revealed markedly increased apathy, disinhibition, and reduction of executive abilities. At month 14 he became increasingly psychotic and was observed to hallucinate visually. He was now unable to imitate alternating hand movements or perform anti-saccades, whereas his other motor, oculomotor, and speech functions were still unremarkable.

\section{DISCUSSION}

The predominant deficit in this case of FD was a severe impairment of executive functions, mainly including planning, problem solving, and set shifting capacities, but also flexibility and divergent thinking. Furthermore, episodic memory and attentional functions were also grossly compromised. In contrast, basic and higher motor functions, language and basic perceptual abilities remained unimpaired. In addition, the patient experienced a marked personality change with apathy, intermittent disinhibition, irritability, aggression, compulsive features, and anxiety. The functional imaging correlate of the basal ganglia calcification was a regional hypometabolism of the basal ganglia and frontal brain as shown by FDG-PET, whereas brain regions located more posteriorly appeared to have normal metabolism. Hypometabolism in both frontal lobes was extensive and included dorsolateral, ventromedial, and orbitofrontal areas.

The origin of this patient's disorder is not clear. Combinations of FD with other neurological conditions have been reported, among them corticobasal degeneration, ${ }^{12}$ mitochondrial encephalopathy, central nervous system lupus, motor neurone disease, Alzheimer's disease, or frontal lobe dementia. ${ }^{2}$ Although none of these disorders can be ruled out completely without histopathology, the basal ganglia lesions remain a crucial finding. The concept of frontal-subcortical

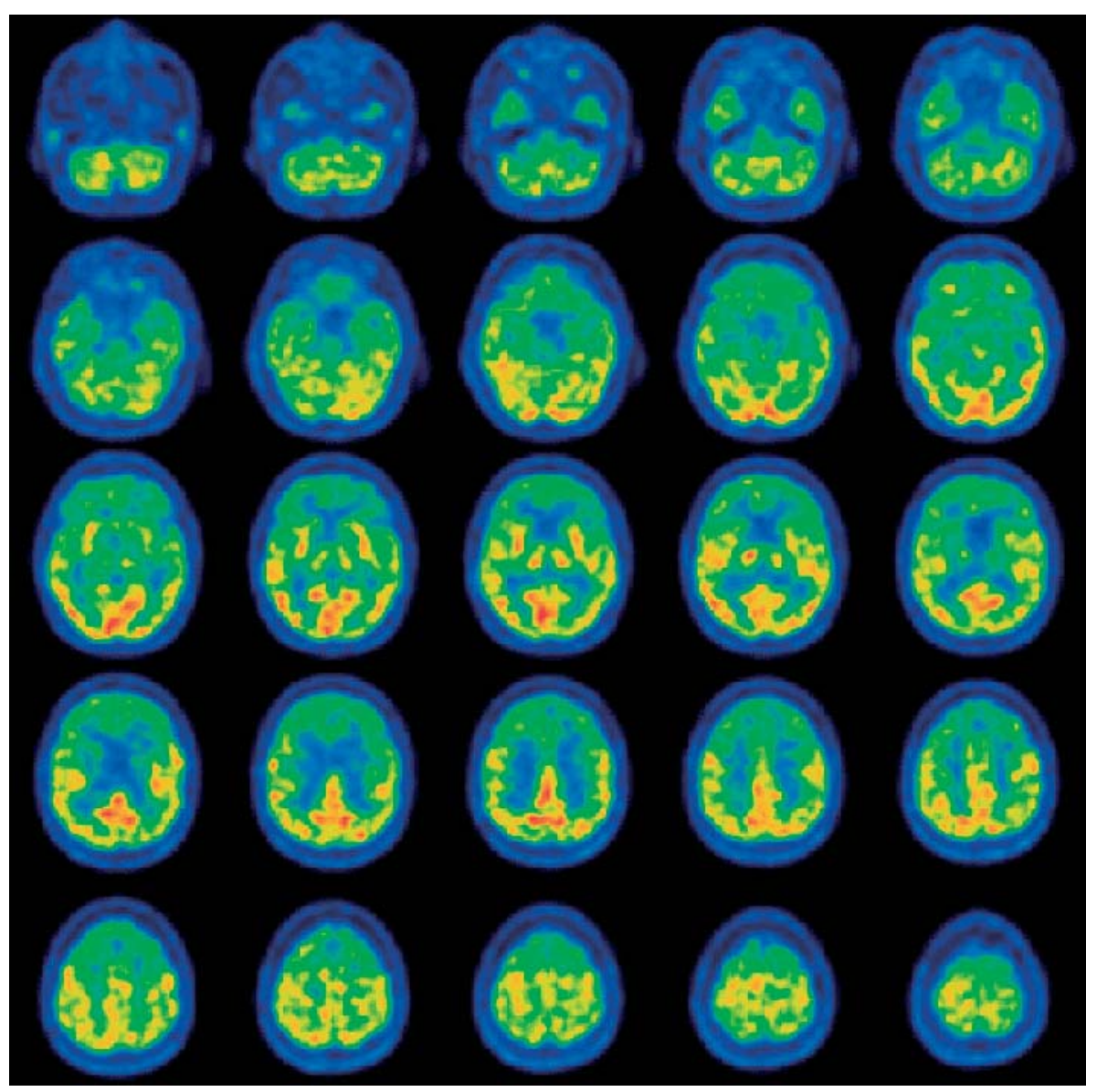

Figure 1 F18-FDG PET brain scan (transverse slices). Significant reduction of glucose metabolism are evident in the basal ganglia and frontal lobes, particularly the dorsolateral, orbitofrontal, and ventromedial cortex. No significant hypometabolism is seen in the temporal or parietal cortex. 
circuits, ${ }^{13}$ a system of loops linking the basal ganglia and frontal brain regions provides a useful, but still hypothetical framework for the interpretation of cognitive and psychiatric disorders in patients with basal ganglia disorders. According to this hypothesis, impairment of executive functions in basal ganglia disorders results from a disruption of the dorsolateral fronto-striatal circuit. ${ }^{14}$ Loss of executive control is also known to produce memory deficits due to loss of organisational, strategic, and monitoring skills necessary for such tasks as list learning, and may follow disruption of the dorsolateral-striatal circuit both at the cortical ${ }^{15}$ and the basal ganglial level. ${ }^{16}$ Attentional dysfunction, including impairment of alertness and of sustained, directed, and divided attention is a frequent finding following dorsolateral and ventromedial prefrontal lesions. ${ }^{17-20}$ Further regions of hypometabolism in our patient were found in orbitofrontal and anterior cingulate areas, which are important parts of the limbic system. Lesions in these areas or the connecting lateral orbitofrontal circuit produce socially inappropriate behaviour, impulsivity, obsessive-compulsive and mood disorders, and personality change ${ }^{14} 21-23$, all of which were part of the behavioural disorder of the present case. These impairments stand in contrast to well preserved cognitive functions such as language or praxis, which are functionally linked with temporal and parietal brain areas showing normal metabolism. Similarly, the patient did not show any of the frequently encountered motor deficits of FD, such as parkinsonism, paresis, spasticity, or gait disorder, ${ }^{2}$ suggesting that his "motor loops" were intact. Only oculomotor impairment was observed during advanced disease stages.

The present case illustrates that FD may present neurologically "asymptomatic" — that is, lacking movement disorders, seizures, or stroke-like events, ${ }^{2}$ but with pronounced, rapidly progressive cognitive and behavioural abnormalities. A clear cut correslation was found between neuropsychiatric features and areas of focal hypometabolism as mapped by FDG-PET. As in other basal ganglia disorders, functional imaging and the concept of cortico-subcortical loops provide a promising account of the underlying mechanism. Although slightly different patterns of hypometabolism have also been found, ${ }^{56}$ FD appears to be a basal ganglia disorder associated with dysfunction in predominantly frontal cortical areas.

\footnotetext{
Authors' affiliations

T Benke, E Karner, K Seppi, M Delazer, University Clinic of Neurology, Innsbruck, Austria

J Marksteiner, University Clinics of Psychiatry and Nuclear Medicine, Innsbruck, Austria

E Donnemiller, University Clinic of Nuclear Medicine, Innsbruck, Austria Competing interests: none declared

Correspondence to: $\operatorname{Dr} T$ Benke, Klinik für Neurologie Innsbruck, Anichstr. 35, A-6002 Innsbruck, Austria; thomas.benke@uibk.ac.at
}

Received 26 May 2003

Revised 19 August 2003

Accepted 16 September 2003

\section{REFERENCES}

1 Lopez-Villegas D, Kulisevsky J, Deus J, et al. Neuropsychological alterations in patients with computed tomography-detected basal ganglia calcification. Arch Neurol 1996;53:251-6.

2 Lauterbach EC, Cummings JL, Duffy J, et al. Neuropsychiatric correlates and treatment of lenticulostriatal diseases: a review of the literature and overview of research opportunities in Huntington's, Wilson's, and Fahr's diseases. A report of the ANPA Committee on Research. American Neuropsychiatric Association. J Neuropsychiatry Clin Neurosci 1998;10:249-66.

3 Forstl H, Krumm B, Eden S, et al. Neurological disorders in 166 patients with basal ganglia calcification: a statistical evaluation. J Neurol 1992;239:36-8.

4 Manyam BV, Bhatt MH, Moore WD, et al. Bilateral striopallidodentate calcinosis: cerebrospinal fluid, imaging, and electrophysiological studies. Ann Neurol 1992;31:379-84.

5 Uygur GA, Liu Y, Hellman RS, et al. Evaluation of regional cerebral blood flow in massive intracerebral calcifications. J Nucl Med 1995;36:610-12.

6 Hempel A, Henze M, Berghoff C, et al. PET findings and neuropsychological deficits in a case of Fahr's disease. Psychiatry Res 2001;108:133-40.

7 Zimmermann P, Fimm B. Testbatterie zur Aufmerksamkeitsprüfung. Herzogenath: Psytest, 1993.

8 Wilson BA, Alderman N, Burgess PW, et al. Behavioural assessment of the dysexecutive syndrome. Bury St Edmunds: Thames Valley Test Company, 1996.

9 Flowers KA, Robertson C. The effect of Parkinson's disease on the ability to maintain a mental set. J Neurol Neurosurg Psychiatry 1985;48:517-29.

10 Cummings JL, Mega M, Gray K, et al. The Neuropsychiatric Inventory: comprehensive assessment of psychopathology in dementia. Neurology 1994;44:2308-14.

11 Grace J, Malloy PF. Frontal systems behavioral scale. Lutz, FL: Psychological Assessment Resources, 2001.

12 Warren JD, Mummery CJ, Al Din AS, et al. Corticobasal degeneration syndrome with basal ganglia calcification: Fahr's disease as a corticobasal look-alike? Mov Disord 2002;17:563-7.

13 Alexander GE, DeLong MR, Strick PL. Parallel organization of functionally segregated circuits linking basal ganglia and cortex. Annu Rev Neurosci 1986;9:357-81.

14 Mega MS, Cummings JL. Frontal-subcortical circuits and neuropsychiatric disorders. J Neuropsychiatry Clin Neurosci 1994;6:358-70.

15 Alexander MP, Stuss DT, Fansabedian N. California Verbal Learning Test: performance by patients with focal frontal and non-frontal lesions. Brain 2003; 126: 1493-503.

16 Benke T, Delazer M, Bartha L, et al. Basal ganglia lesions and the theory of fronto-subcortical loops: neuropsychological findings in two patients with left caudate lesions. Neurocase 2003;9:70-85.

17 Stuss DT, Binns MA, Murphy KJ, et al. Dissociations within the anterior attentional system: effects of task complexity and irrelevant information on reaction time speed and accuracy. Neuropsychology 2002;16:500-13.

18 Daffner KR, Mesulam MM, Holcomb PJ, et al. Disruption of attention to novel events after frontal lobe injury in humans. I Neurol Neurosurg Psychiatry 2000;68: 18-24.

19 Sturm W, Willmes K. On the functional neuroanatomy of intrinsic and phasic alertness. Neuroimage 2001;14:S76-S84.

20 Godefroy O, Lhullier C, Rousseaux M. Non-spatial attention disorders in patients with frontal or posterior brain damage. Brain 1996;119(Pt 1): 191-202.

21 Assal F, Cummings JL. Neuropsychiatric symptoms in the dementias. Curr Opin Neurol 2002;15:445-50.

22 Miller BL, Darby A, Benson DF, et al. Aggressive, socially disruptive and antisocial behaviour associated with fronto-temporal dementia. Br J Psychiatry 1997; 170: 150-4

23 Blair RJ. Neurocognitive models of aggression, the antisocial personality disorders, and psychopathy. I Neurol Neurosurg Psychiatry 2001;71:727-31. 